\title{
Bioethical perspective of palliative care
}

Keywords: bioethics, scope, palliative care, technical experience, terminal diseases

\section{Opinion}

As a discipline Bioethics has taught us that in the current era issues and problems can't, and should not, be addressed from a single point of view. Reality shows that problems are complex, and therefore require a multidimensional approach that integrates various disciplines in order to be understood and resolved as a whole.

Considering this, the bioethical perspective of palliative care, demands that we open our scope of view in order to evaluate its impact and result, going beyond what symptoms, technical experience and professional training has taught us. In fact, palliative care is much more than just therapeutic actions aimed at mitigating pain in the face of chronic pathologies, irreversible states or terminal diseases. In the final stages where the intention of medicine is not to cure but to palliate. Palliative should care not to symptoms, but to the person and it's environment. This is why attention must focus not only on the clinical and physical manifestations of suffering, but also on the psychic and social effects of the disease. Accordingly, those who dedicate their professional life to palliative care need more than just technical abilities, but additionally social and communicational skills. An attitude of permanent empathy towards the reality of the others should be mandatory. Empathy is to look at someone else and be able to reflect on what the other is experiencing, knowing that what they requires could go beyond my specialized training; recognizing that they also needs my attention, contention and recognition as another person, and as autonomous being.

Furthermore, from a bioethical perspective, palliative care should also focus on the assistance of caregivers. Even though they play a critical role in the care of the chronic and terminally ill patient, they often become overshadowed by the burden of the disease. They tend to neglect their own personal and professional life, and become socially undervalued for their many times, unpaid work. Studies show that commonly these caregivers are woman; a mother, daughter, sister, or grandmother; who postpones her life in favor of that of the patient. Palliative care professionals must ask themselves: who is taking care of these caregivers?

Under this holistic view, palliative care poses a challenge to society as a whole; to stand up and take a position in the face of human pain and considering our current capacity to respond to it. We are compelled to socially value that a space should be granted to technique and also to autonomy. Palliative care relates to irreversible situations, to incurable diseases, and decisions that could affect the final moments of human life. In fact, palliative care confronts us to uncertain situations such as rejection of treatments, therapeutic boldness or even euthanasia itself.

All this issues, even though they implicate the patient, also impact its community. That is why palliative care should be also approached from a social perspective, based on agreements (consensus) and with a clear and timely disposition to establish the "rules of the game".
Volume 2 Issue 3 - 2018

\author{
Blanca Bórquez Polloni \\ Researcher, Bioethics and Law Observatory UNESCO Chair in \\ Bioethics, University of Barcelona, Spain
}

Correspondence: Blanca Bórquez Polloni, Researcher, Bioethics and Law Observatory UNESCO Chair in Bioethics, University of Barcelona, Ave. Diagonal 684, Faculty of Law, 08034 Barcelona, Spain, Tel (+34) 9340345 46,

Email blancabp@hotmail.com

Received: April 29, 2018 | Published: June 042018

This statement, to which palliative care invites, opens the opportunity for debating and deliberating on matters that are especially sensitive for social and legal validating bodies: the parliaments. However, opening these spaces is never easy, especially in conservative cultures that are shut to the option of recognizing the plurality of moral options of other members of society. That is why we must not forget that palliative care should be offered considering the diversity of geographical, social, cultural and economic realities, all over the world. Thus, the problems and challenges of answering these issues are complex and require willingness and eagerness to put these matters on the table. But it also entails empathy, in order to see others and their reality, and those decisions should not be blinded by personal convictions. When opening the legislative space to discuss the scope of palliative care, is important to consider its funding, universality, limits and also discuss the autonomy of patients. Nevertheless, this should not be a source of fear of slippery slopes or unsuspected extensions; on the contrary, it is an act of courage to empathize with others through dialogue and deliberation. Until these social conditions are created, it is necessary to reinforce to those who work in palliative care the idea that, above all, patients should not be harmed and this means treating them with respect for their dignity, considering their personal reality, avoiding cruel unnecessary procedures, and not expecting intrepid behaviors from those who suffers the disease or the ones that take care of them. As stated in the "Loi concerned de les soins de vie" of the National Assembly of Quebec (Canada), at the end of life people must be guaranteed health care that respects their dignity and autonomy, and that it is aimed to prevent and mitigate their suffering. The bioethical perspective of palliative care shows that we face an area of health with a high social content and that we all have the right to live and die in freedom.

\section{Acknowledgements}

None.

\section{Conflict of interest}

The author declares no conflict of interest. 